\title{
A Research on the Health Promoting Effect of Taijiquan
}

\author{
Zhao Fatian ${ }^{1, ~ *, ~ L i ~ L e i ~}{ }^{1}$, Zhang Le ${ }^{2}$ \\ ${ }^{1}$ PE Institute, Shandong Normal University, Jinan, China \\ ${ }^{2}$ PE Group, Shandong University Affiliated Primary School, Jinan, China
}

Email address:

1152079682@qq.com (Zhao Fatian),3211434246@qq.com (Li Lei),276976624@qq.com (Zhang Le)

${ }^{*}$ Corresponding author

\section{To cite this article:}

Zhao Fatian, Li Lei, Zhang Le. A Research on the Health Promoting Effect of Taijiquan. American Journal of Sports Science.

Vol. 7, No. 4, 2019, pp. 159-163. doi: 10.11648/j.ajss.20190704.15

Received: September 20, 2019; Accepted: October 31, 2019; Published: November 6, 2019

\begin{abstract}
Health is an issue that every person should pay attention to. Chinese President Xi Jinping also emphasized in his speech that, staying health is a must in terms of becoming an all-round person, it is regarded as the foundation for economic and social development. Besides, it is also a crucial symbol for national and state prosperity, as well as a common pursuit of the masses. Through literature review and expert interview, this paper gives an in-depth explanation on the cultural connotation behind "practicing both inside and outside of the body, movement based on one's own mind" in Taijiquan (Taijiquan, a kind of traditional Chinese slow movement). Besides, this paper also studies on Taijiquan's movement which featured in slow and smooth, light and flexible, harmony and balance between dynamic and static, and its promoting effect on health. It is concluded that Taijiquan is effective in terms of supporting the general wellbeing of nervous system, cardiovascular system, respiratory system, immune system, digestive system and sports system. The effect is especially significant for forming healthy positive emotions, cultivating healthy personality and improving the ability of social adaptation.
\end{abstract}

Keywords: Health, Taijiquan, Health Promotion, Effect

\section{Introdution}

The advancement of science and technology has gradually replaced the primary and secondary industries in the tertiary industry of China's economic sector. The emergence of mental labor has gradually replaced physical labor. The lack of exercise, complex interpersonal relationships, and social pressure have led to a decline in physical fitness and fatigue. A "sub-health" state of symptoms such as emotional uneasiness and neurasthenia. As the crystallization of the wisdom of the Chinese nation, Taijiquan follows the philosophical connotation of the unity of nature and man, the complementarity of yin and yang, and the harmonious development. It pays attention to the closeness of mind, intentional guidance, gas-guiding ideas, breathing and movement. Cooperate, highlighting the static body, the head straight neck, the shoulders and the elbows, the chest and the back, the tail squat, the loose waist and the body and the essentials, emphasizing the trend, the continuous, the intention less use of the frame requirements. To practice Taijiquan, we must strictly follow the "form-like" premise of correctness, and then carry out the understanding and understanding of the connotation of the connotation, and finally reach the "likeness" realm of swaying and showing the charm. In the cultivation process gradually gain the ability of health care and society adaption.

\section{Taijiquan's Health Promotion Effect on Body Physiology and Function System}

\subsection{Taijiquan's Health Promotion Effect on the Nervous System}

Taijiquan requires "the heart is quiet, the mind is concentrated" and the "heart and body loose" is expressed as meditation, upward Baihui, keep balance and comfortable, backward Mingmen, body relax, breathing natural and quiet. This is not only need bones, muscles and internal organs relax, it is also requiring mental relax, with no distracting thoughts. "Concentration of thoughts" is manifested by the operation of the meridian atmosphere with ideas. The meridian atmosphere is generated by Dantian [1]. It passes through the conduction 
of the two branches, we call them Ren and Du, and finally reaches the limbs. The limbs will have the feeling of heat, swelling and hemp; On the basis of the "inner triad" and the combination of the hand and the foot, the elbow and the knee, the shoulder and the fit, and then inject the idea into the need to the completed action, the intention is to follow. For example: when starting up, you must first have a shoulder, elbows, loose waist and knees, buttocks cannot protrude, the center of gravity falls in the middle of the legs, the fingers naturally bend slightly, the arms fall to the knees of the body The movements are coordinated and the basic movements of the imagination, the body followed by the idea to do [2]. Guided movements can also regulate breathing, promote the coordination of mind, breathing, and movement, and achieve the realm of casual movement. During the process of mind-taking, the cerebral cortex and central nervous system have been well trained and can process and process various kinds of information introduced from the outside, so that the human body can make a complete overall response. Firstly, the central nervous system issues low frequency and relaxation instructions instead of stress, depression and other information, which can improve mental health and adjust the balance between the nervous system and other systems [3]; secondly, the excitability of the vague nerve increases, and the plant is enhanced. The self-control ability of sexual nerves and organ activities can prevent various diseases caused by vegetative neurological disorders common in modern people [4].

\subsection{Taijiquan's Health Promotion Effect on the Cardiovascular System}

Taijiquan requires "the body is loose, natural, soft, slow, tangible and continuous" "Body pine is natural, soft and slow" is characterized by relaxation in the Taijiquan exercise, relaxation is the driving of the mind, not loose, negative, but also accompanied by rhythmic tension, is positive. Therefore, the role of relaxation is: First, it can promote central nervous system function perfect, many neurons gathered in the central nervous system have functions to regulate the cardiovascular system. For example: medullary cardiovascular center, hypothalamus and cerebral cortex. Second, causing reflex relaxation of the blood vessel wall, synergistically opening many capillary networks that are normally open and rotated, and telangiectasia, which improves the blood circulation including microcirculation, reduces the peripheral resistance of blood vessels, and causes blood pressure to drop [5]. "False and real transformation, continuous and continuous" is characterized by the need to be light and round, and the trend is accompanied by rhythm. The rhythm of the tension and the virtual reality makes the muscles and blood vessels in the body rhythmically squeeze and stretch, which stimulates the blood flow around the blood vessel and the blood vessel inside the blood vessel promotes the blood flow per unit time and the elasticity of the blood vessel is enhanced. Its role is as follows: 1. The blood volume of the venous return to the heart per unit time increases, and the ventricular volume increases during the end of the heart. During the ventricular diastolic period, venous blood continuously flows into the ventricle through the atrium to make it fill. When filling to a certain extent, the ventricular muscle is stretched, and the stretch stimulation of the ventricular muscle can increase the contraction force of the heart muscle and increase the stroke output of the heart [6]. Second, the blood flow through each organ increases, scouring the cholesterol and low-density lipoprotein attached to the inner wall of the blood vessel, reducing the incidence of high blood pressure, high blood lipids and high blood sugar "three highs". Therefore, Tai Chi exercise contributes to the improvement of cardiovascular function.

\subsection{Taijiquan's Health Promotion Effect on the Respiratory System}

Taijiquan requires "slim, uniform, deep, long" abdominal breathing, which stimulates the diaphragm, abdominal muscles and intercostal muscles, promotes contraction and relaxation of respiratory muscles, and facilitates the expansion and contraction of the thoracic rhythm. The retractive force is enhanced, the motive force of lung ventilation is enhanced, and the negative pressure in the chest is increased, which not only maintains the expansion state of the lung, but also facilitates the gas exchange of the alveoli. According to physiology, due to the existence of anatomical ineffective cavity, the alveolar ventilation in deep and slow breathing is more than that in shallow and fast breathing, which improves the ventilation function of the alveoli, thus ensuring more oxygen intake and promoting the body metabolism [7]. Taijiquan is an aerobic exercise with moderate intensity. The energy consumed in exercise is completely supplied in the body's aerobic metabolism. Therefore, Tai Chi exercise is safe for people's respiratory system. When practicing Taijiquan, it requires breathing and movement to cooperate with each other It is based on the premise of not destroying the natural breathing and the rhythm of movement. It does not require deliberate and mechanical cooperation and takes the deep reverse abdominal breathing as much as possible. The tension between the lungs and the thorax is increased, the lung capacity is increased, the contact area between the alveoli and the capillary wall is increased, and the oxygen and carbon dioxide diffusion ability are enhanced [8].

\subsection{Taijiquan's Health Promotion Effect on the Immune System}

Taijiquan requires "being light and working and slowing down". The movements are like flowing water, flowing and free. "There is a whole body moving, the whole body moves and there is no movement, the whole body is quiet and there is no static, its static movement, although it is still quiet, the technical characteristics of Taijiquan determine Taijiquan as a moderately exercised, slightly sweaty fitness activity. Physiology has shown that moderate, moderate-intensity exercise can increase the number and function of immune cells. On the one hand, due to moderate exercise, the activity of the sympathetic nerves is reduced, and the body's sensitivity to stress is reduced, resulting in catecholamines and 
cortisol. The secretion of hormones is reduced, which is manifested by enhanced immune function. On the other hand, long-term adherence to moderate intensity load of Tai Chi practice will cause lymphocytes to be repeatedly exposed to other inhibitory hormones, and the number and sensitivity of lymphocyte surface hormone receptors will decrease, so that lymphocytes do not act on hormones. Sensitive, also expressed as the body's immune function is enhanced [9].

Epidemiological surveys have shown that moderate exercise can increase the activity of certain enzymes, destroy the predisposing factors that produce cancer, improve immune function, increase systemic immunity and the number and function of T cells and B cells, and increase the number and ability of killer cells. For example, the activity of $\mathrm{K}$ cells in athletes' resting state is higher than that of ordinary people, so exercise can prevent cancer [10]. Moderate, moderate-intensity exercise can also increase the content and concentration of immune molecules. Studies have shown that moderate exercise can improve the body's antibody level and improve immune function. The level of immunoglobulin A secreted by the respiratory tract directly affects the resistance of the respiratory mucosa to pathogens. The two are positively correlated, Huang Qiping [11]. A group of 30 female college students who participated in the simplified Taijiquan exercise conducted research and concluded that adhering to the moderate amount of Taijiquan aerobic exercise can increase serum Ig A content and enhance the body's humoral immune response, thereby improving the ability of antigen pathogen infection. Liu Yanhuan believe that the level of serum immunoglobulin Ig A, Ig G can be increased, and the upper respiratory tract can be shortened after 3 times a week and 5 times a week [12]. The duration of infection indicates that Tai Chi exercise can improve the body's immunity and enhance the body's ability to resist upper respiratory tract infections.

\subsection{Taijiquan's Health Promotion Effect on the Digestive System}

Taijiquan requires "take waist as axis, use waist lead limb, rotate Dantian inside, fold chest and waist", and Taijiquan movement uses the rhythmic abdominal breathing and the belt limb, the thoracolumbar folding movement to drive the stomach, intestines and liver in the digestive system. The systolic and diastolic organs of the spleen and the diastolic organs, and the organ peristalsis are enhanced, which promotes the flow of blood in the abdominal cavity, increases the blood circulation in the stomach, and improves the digestion and absorption capacity of the digestive organs, which is a physical digestion. The mechanical movement of the digestive organs is increased, stimulating the stomach to produce stomach acid, stimulating the intestinal gland to produce intestinal fluid, stimulating the pancreas to produce pancreatic juice, stimulating the gallbladder to produce bile, and promoting the digestion of food through the corresponding chemical liquid secreted by the digestive organs, which belongs to chemical digestion. Taijiquan also requires "tongue to the upper jaw", which is conducive to oral hygiene, and then swallow saliva. Saliva contains mucin, mucopolysaccharide, salivary amylase, lysozyme, immunoglobulin blood type substance, free amino acid, etc., which is beneficial to digestion. Taijiquan has a good regulation function on the digestive system and has a good improvement on constipation symptoms.

\subsection{Taijiquan's Health Promotion Effect on Exercise System}

Taijiquan requires "moving and quiet, real and virtual transformation, footwork steady". In practicing boxing, it is necessary to ensure the movement of the movement and maintain the balance of the body. Emphasize the movement of the trunk and limbs with the waist, so that the trunk and limbs can be carried out. There is a right overall movement on the left, so that the cycle of recurrence will stimulate the waist and limbs, enhance the flexibility, coordination and strength of the bones, bones and skeletal muscles, so that the joints and muscles of the human body are both elastic. It also has the power to promote the stability of muscles and bones. Taijiquan is the key to walking. In the boxing, it is said that "the steps are unstable and the boxing is chaotic", and it is required that "the width of the two feet is moderate, the reality is clear and clear, the rise and fall should be soft, the angle of the foot should be accurate, the size of the step should be proper, and the foot should be taken. The arc should be stable, which increases the strength of the buttocks and quadriceps, enhances the load capacity that the knee joint can withstand, and increases the range of ankle joint activity. Muscle strain and joint sprains caused by insufficient strength of the lower limbs and insufficient joint movement are avoided. Studies have shown that when practicing Taijiquan, it can also promote the synthesis of protein in the body, prevent the degenerative changes of muscle atrophy, reduce its aging rate, maintain and improve the toughness of joint ligaments and joint flexibility, and effectively prevent bone loss. Promotes calcium absorption and prevents osteoporosis [12]. Long-term adherence to practice Taijiquan not only improves the flexion and compression resistance of the bone, but also helps the elderly to improve their "self-awareness", allowing them to be more cautious in their daily lives, doing something within their power, and preventing fall.

\section{Taijiquan Promotes Mental Health}

\subsection{The Promotion Effect of Taijiquan on Healthy Positive Emotions}

Taijiquan requires "be modest, no desire", which makes people in a state of quietness, looseness, peace, carefreeness, and no distraction, forgetting the pressure and exhaustion, trouble and pain in life. Thereby feeling the beauty of life, sensing the joy, optimism, happiness, pride, satisfaction, love, interest, etc. brought by life, forming a positive and healthy mentality, eliminating negative emotions such as sadness, anxiety and depression. Even if the living environment is harsh and the interpersonal relationship is complicated, it can be done without urgency and confrontation with a bold, 
optimistic and cheerful attitude towards life. It has been reported that individuals who have been practicing Taijiquan for a long time can maintain a relatively good awakening state of the brain function - "Tai Chi state". In this state, the frequency of the brain waves is ordered, synchronized, and the spectrum energy is increased. It is a dominant position, which increases the secretion of substances in the caffeine and catecholamines and promotes the body to maintain a pleasant state [13]. In addition, adverse emotions are mainly caused by the left cerebral hemisphere, and the right cerebral hemisphere is the area where positive emotions are generated. Tai Chi practice can gradually suppress the left cerebral hemisphere of the practitioner, excite the right hemisphere, and finally make the right cerebral hemisphere replaces the left to gain dominance, thereby reducing the individual's negative emotional experience, increasing positive emotional experience and improving mental health [14].

\subsection{The Promotion Effect of Taijiquan on the Cultivation of Sound Personality}

Taijiquan requires "Dog day summer training, winter training three nine", which means no matter how cold or hot, should insist on physical exercise. Trainer should insist on getting up early, rain or shine, helping people develop perseverance, and change the lazy, procrastination. as Taijiquan theory said: "Hands move with your heart, you can change with your steps, and you will be able to sink when you turn around." "The joints are not loose; the softness does not come." "Fist is not invincible to the law, the law is not worthy of the merits" "from the familiar and gradually understand the power, by the strength and order and the gods" and so on, and the connotation of the self-existing practice. It is said that the practice of Taijiquan not only remembers the complicated routines, but also pays attention to the acquisition of the exercises and verifies the philosophical truth of "Taijiquan does not go out for ten years." In the process of cultivation, not only the people's will be honed, but also the overall quality and cultivation of the people. Taijiquan is bred by China's rich traditional culture, including Confucianism's "Benevolence, Righteousness, Courtesy, Wisdom, and Faith", Taoist's "Tao Law Nature", Buddhism's "Compassion and Tolerance" Principle of Being a Person, Philosophy of Being, and Soundness Personality has a significant effect on the quality of optimism, confidence, and courage.

\subsection{The Promotion Effect of Taijiquan on Improving People's Social Adaptability}

Taijiquan requires "smooth peace, calmness, quietness, and static movement", which reduces the sensitivity to self-emotion and external environment interference, thereby improving the psychology of the practitioners and improving their ability to calm down. Enhance the level of harmony with people. Taijiquan has the function of benefit group. Every morning or evening, group exercises are conducted along the square and the woods. The practitioners exchange ideas with other people of all walks of life who have common interests, learn from each other and explore their experiences. Can cultivate people's communicative ability and improve social adaptability.

\section{Summary}

As a representative of Chinese excellent culture, Taijiquan requires practitioners to "relax body, keep mind and body calm and concentrate, when practice Taijiquan, keep all movements soft, slow, continuous, gentle. Take waist as axis, use waist lead limb, rotate Dantian inside, fold chest and waist Memorize key point of practice is moving and quiet, real and virtual transformation, footwork steady, no matter how cold in winter and how hot in summer, just keep Taijiquan training. The basic normative requirements will have a health promotion effect on the body's physiological function system, mental health and social adaptability, promote human health, and facilitate the implementation of the "Healthy China 2030" program.

\section{References}

[1] Xi Jinping. Outline of "Healthy China 2030". Political Bureau of the CPC Central Committee. 2016. 8. 26.

[2] Cai Zhonglin, Zhou Zhihua. Martial Arts [M]. Beijing: Higher Education Press, 2009, (6): 147.

[3] Yu Dinghai. Analysis of the current status of health promotion research in Taijiquan exercise [J]. Zhongzhou Sports. Shaolin and Taiji, 2011, (12): 33-35.

[4] Fan Xuefeng, Yao Yaxing. Analysis of the fitness value of Taijiquan [J]. Fighting $\cdot$ martial arts, 2014, (6): 51-53.

[5] Zheng Rui, Zhou Yuanchao, Xu Yidan. Study on Taijiquan exercise to improve the status of chronic diseases in middle-aged and elderly people [J]. Fighting - martial arts, 2012, (4): 41-43.

[6] Wang Bubiao, Hua Ming. Sports Physiology [M]. Beijing: Higher Education Press, 2011: 70.

[7] Sang Quanxi. Exploring the Techniques and Methods of Taijiquan Fitness [M]. Beijing: China Book Publishing House, 2010: 17.

[8] Zhang Zhilei, Zhu Dong. Taijiquan intervention exercise program for rehabilitation of heart and lung chronic diseases $[\mathrm{J}]$ Chinese Journal of Gerontology, 2019, 07.

[9] Wang Bubiao, Hua Ming. Sports Physiology [M]. Beijing: Higher Education Press, 2011: 190.

[10] Huang Yuping. Effects of Taijiquan on Enhancing Serum Total Complement Activity of Female College Students [J]. Journal of Physical Education, 2006, 13, (2): 70.

[11] Liu Yanhuan. The effect of Taijiquan on immunoglobulin in female college students and its relationship with upper respiratory tract infection [J]. Journal of Beijing Sport University, 2005, 28. (8): 1089.

[12] Sang Quanxi. Exploring the skills and methods of Taijiquan fitness. [M] Beijing: China Book Press, 2010: 25. 
[13] Chen Qinghe, Li Yigang, Zheng Yongcheng et al. The role of Taijiquan in improving the mental health status of trainers [J]. Chinese Journal of Clinical Rehabilitation, 2006, 10, (43): 40-42.
[14] Wei Dexiang. The Relationship between Taijiquan Exercise and Mental Health: A Meta-analysis Study [J]. Journal of Fujian Normal University (Natural Science Edition), 2011, 27, (5): 111-116. 